\title{
RELAXAÇÃO DE TENSÕES DE POLÍMEROS - MODELAÇÃO DE MATERIAIS NÃO-LINEARES
}

\author{
MODELLING OF NON-LINEAR MATERIALS - POLYMER STRESS \\ RELAXATION
}

\section{RELAJACIÓN DEL ESTRÉS POLIMÉRICO/ DE POLÍMEROS - MODELADO DE MATERIALES NO LINEALES}

\author{
José Reinas dos Santos André (jandre@ipg.pt)* \\ José Joaquim C. Cruz Pinto (jj.cruz.pinto@ua.pt)**
}

\section{RESUMO}

Neste trabalho é aplicado um modelo, desenvolvido previamente pelos autores, que permite prever a relaxação de tensões de polímeros amorfos e semi-cristalinos, que contempla a interligação da temperatura e a deformação. As alterações induzidas pela deformação foram investigadas através de ensaios de relaxação não linear de tensões em dois polímeros a três temperaturas. O modelo é sensível a diferentes estados iniciais do material, resultantes da variação da orientação molecular, bem como de distintos níveis de envelhecimento, e a partir de dados experimentais do módulo de relaxação de tensões prevê para o poli(metacrilato de metilo) - PMMA e o poli(tereftalato de etileno) - PET os valores da temperatura crossover, $T_{c}$, da energia de ativação mínima, $E_{\alpha, 1}$, para além do patamar dos módulo de relaxação instantáneo, $E_{0}$, e o patamar do módulo para tempos longos, $E_{\infty}$, de acordo com os valores encontrados na literatura.

Palavras Chave: relaxação de tensões, poli(metacrilato de metilo), PMMA, poli(etileno tereftalato); PET, espetro de relaxação.

\section{ABSTRACT}

In this study, a model previously developed by the authors is applied, one which allows for prediction of stress relaxation of amorphous and semi-crystalline polymers and which contemplates the interconnection of temperature and deformation. Deformation-induced changes were investigated by non-linear stress relaxation tests on two polymers at three temperatures. The model is sensitive to the different initial states of the material, resulting from varying molecular orientation as well as different age levels, and from experimental data of the stress relaxation module predicts the poly (methyl methacrylate) - PMMA and poly (ethylene terephthalate) - PET the values of crossover temperature, $T C$, minimum activation energy, $E_{\alpha, 1}$, in addition to the instantaneous relaxation modulus plateau, $E_{0}$, and the long-time modulus plateau, $E_{\infty}$, according to the values found in expert literature. Keywords: stress relaxation, poly(methylmethacrylate), PMMA, poly(ethylene terephthalate), PET, relaxation spectrum. 


\section{RESUMEN}

En este trabajo se aplica un modelo, desarrollado previamente por los autores, que permite predecir de la relajación del estrés de polímeros amorfos y semicristalinos, que contempla la interconexión de la temperatura y la deformación. Los cambios inducidos por deformación se investigaron mediante pruebas de relajación de estrés no lineal en dos polímeros a tres temperaturas. El model+++o es sensible a los diferentes estados iniciales del material, como resultado de la orientación molecular variable, así como a diferentes niveles de envejecimiento y, a partir de los de los datos experimentales del módulo de relajación del estrés, se predice para el poli (metacrilato de metilo) PMMA y el poli (tereftalato de etileno) los valores de la temperatura de crossover, $T_{c}$, energía de activación mínima, $E_{\alpha, 1}$, además del módulo de relajación instantánea, $E_{0}$ y el módulo de relajación para tiempos largos, $E_{\infty}$, de acuerdo con los valores encontrados en la literatura.

Palabras-clave: relajación de tensiones, poli (metacrilato de metilo), PMMA, poli (tereftalato de etileno), PET, espectro de relajación.

$$
\begin{aligned}
& \text { *Professor Coordenador da Escola Superior de Tecnologia e Gestão do } \\
& \text { Instituto Politécnico da Guarda. Membro da Unidade de Investigação } \\
& \text { para o Desenvolvimento do Interior - UDI. } \\
& \text { ** Professor Catedrático Aposentado, Departamento de } \\
& \text { Química/CICECO, Universidade de Aveiro. }
\end{aligned}
$$

Submitted:28 ${ }^{\text {th }}$ February 2020

Accepted: $25^{\text {th }}$ October 2020 


\section{INTRODUÇÃO}

Num ensaio de relaxação de tensões é aplicada de forma rápida uma deformação no material, que posteriormente se mantém constante, enquanto que a tensão decresce ao longo do tempo. Como é comummente aceite o processo de relaxação de tensões apresenta maior dificuldade de descrição e modelação do que a fluência (aumento gradual da deformação quando o material é sujeito a uma tensão constante), o que justificará investigação mais significativa e avançada neste caso (André 2004; Houshyar, et al., 2005) do que no da relaxação de tensões (André et al., 2014; Findley, et al., 1989).

A particularidade da viscoelasticidade nos materiais poliméricos reside nos complexos mecanismos de conformação molecular que estão na base das deformações macroscópicas observadas, como resultado dos rearranjos dos segmentos das cadeias moleculares, função do tempo, e com uma cinética dependente necessariamente da temperatura a que o material se encontra, como discutido em anteriores trabalhos dos autores (André 2004; André et al., 2014; Cruz Pinto et al., 2016).

Os fundamentos da viscoelasticidade têm sido normalmente inspirados em experimentação, designadamente em ensaios de relaxação de tensões e de fluência, contemplando diferentes padrões de tensão, deformação e temperaturas. É pois possivel uma aproximação experimental à interpretação dos modelos teóricos geralmente propostos, deduzir um conjunto de informações relevantes quanto à física do polímero, quantificar a velocidade e o tipo de interações dos mecanismos conformacionais inerente à estrutura molecular, ou de um ponto de vista mais prático, prever o comportamento dos materiais em serviço em termos de um reduzido número de parâmetros e experiências.

Neste trabalho vamos considerar no processo de relaxação de tensões a inexistência de escoamento viscoso, que será tido em consideração em trabalhos posteriores.

Os modelos apresentados na literatura para interpretar o comportamento de materiais poliméricos a solicitações de relaxação de tensões são essencialmente empíricos ou semiempíricos, não tomando em consideração os mecanismos físicos (moleculares) responsáveis pelo comportamento viscoelástico. Alguns investigadores (Sweeney, et al., 1990) ainda recorrem à aplicação de modelos empíricos, fenomenológicos, baseados em modelos mecânicos que combinam elementos elásticos (de comportamento Hookeano), representados por molas, e elementos viscosos (de comportamento Newtoniano), representados por amortecedores viscosos, ao invés de tentar fisicamente, com base molecular, descrições do comportamento. Diversos trabalhos publicados são meramente teóricos (Kubát, 1979; Hogfors, et al., 1981; Kubát, 1982), enquanto outros são de simulação (Brostow, et al., 1993; Blonsky et al., 1994). No presente estudo, é aplicado um modelo molecular previamente desenvolvido pelos autores (André et al., 2014; Cruz Pinto et al., 2016) com o intuito de descrever a relaxação de tensões de materiais poliméricos, a várias deformações e diferentes temperaturas, com e sem tratamento térmico prévio, obtendo-se previsões teóricas com base em resultados experimentais. 


\section{PARTE EXPERIMENTAL}

\subsection{Material}

Os materiais utilizados neste estudo, um amorfo e outro semi-cristalino, foram, respetivamente, o poli(metacrilato de metilo) (PMMA) e o poli(etileno tereftalato) (PET), ambos disponíveis na forma de folhas com a espessura de $4 \mathrm{~mm}$.

\subsection{Métodos}

Os provetes, com as dimensões de $150 \mathrm{~mm} * 10 \mathrm{~mm} * 4 \mathrm{~mm}$, foram inicialmente cortados com uma serra, posteriormente foram maquinados com uma fresadora de comando numérico, CNC, Luxtronic e, finalmente, polidos com folhas de $\mathrm{SiC}$, sendo o polimento final feito com lixas P1200. A secção transversal de todos os provetes foi medida com paquímetro digital, e posteriormente, antes de se dar início ao ensaio de relaxação de tensões, as dimensões da espessura e da largura foram inseridas no programa.

\subsection{Ensaios de Relaxação de Tensões}

Os ensaios de relaxação de tensões foram realizados numa Máquina de Ensaios Universal Zwick Z100, com uma célula de carga de $2,5 \mathrm{kN}$. As deformações foram medidas com um extensómetro Macro com um curso de leitura de $100 \mathrm{~mm}$. Os dados experimentais da força/deformação vs. tempo foram adquiridos simultaneamente através de um computador pessoal.

Os provetes de PET foram sujeitos a tratamento térmico de tempera: aquecidos a 85 ㄷ C durante 30 minutos, tendo posteriormente sido drasticamente arrefecidos em água a 6 드, durante 30 minutos. As deformações aplicadas nos polímeros foram de 3, 4 e $5 \%$. Os provetes foram previamente condicionados a $23^{\circ} \mathrm{C}$ numa câmara climática, e os testes, em ambos os materiais, foram realizados com uma câmara termostática, às temperaturas de $30^{\circ} \mathrm{C} \mathrm{e} 40^{\circ} \mathrm{C}$, sendo o PMMA testado também a $50^{\circ} \mathrm{C}$. A duração dos ensaios foi de 2,5 horas para o PMMA e de 24 horas para o PET.

\section{MODELO DE RELAXAÇÃO DE TENSÕES}

A principal característica do processo de relaxação de tensões de um material polimérico (longe da rotura) é o decréscimo progressivo da tensão com uma velocidade decrescente, até essa velocidade se tornar nula (tensão constante) ou simplesmente constante (característica de um comportamento viscoso).

Os autores (André et al., 2014; Cruz Pinto et al., 2016), formularam para a gradual redução de tensões, $\sigma(t)$, e o correspondente módulo de relaxação, $E_{r}(t)=\sigma(t) / \varepsilon_{0}$, a expressão matemática, 


$$
\begin{gathered}
\text { E GI T A N I A S C I EN C I A } \\
E_{r}(t)=E_{\infty}+\left(E_{0}-E_{\infty}\right) \frac{\ln t}{\int_{\ln t}^{+\infty} e^{-\left[b \ln \left(\theta / \theta^{*}\right)\right]^{2}} d \ln \theta}=E_{\infty}+\left(E_{0}-E_{\infty}\right) \frac{1+e r f\left[b \ln \left(\frac{\theta^{*}}{t}\right)\right]}{1+e r f\left[b \ln \left(\frac{\theta^{*}}{\theta_{1}}\right)\right]}
\end{gathered}
$$

em que bé proporcional ao inverso do desvio padrão dos vários tempos de relaxação, tal que $b=b_{0} / I n\left(\theta^{*} / \theta_{1}\right)$, onde $\theta_{1}$ é o tempo mínimo de relaxação, e $\theta^{*}$ o médio, com $E_{r}\left(t \leq \theta_{1}\right)=E_{0}$.

Deste modo, para uma dada deformação inicial imposta, $\varepsilon_{0}$, e temperatura $T$, é possível determinar o tempo mínimo de relaxação, $\theta_{1}$, o tempo médio de relaxação, $\theta^{*}$, para além da largura do espectro dos tempos de relaxação, através do parâmetro $b$ (proporcional ao inverso do desvio padrão, $d p$, dos tempos de relaxação, $\left.b=\frac{1}{d p \sqrt{2}}\right)$, e dos valores correspondentes aos patamares do módulo de elasticidade para tempos curtos, $E_{0}$, e tempos longos, $E_{\infty}$.

De acordo com (Cruz Pinto et al., 2016), com base na equação anterior obtém-se o espetro dos tempos de relaxação para o material a várias temperaturas e deformações,

$$
H(\theta)=\frac{b_{0} \sqrt{\pi}}{\left[1+e r f\left(b_{0}\right)\right] \ln \left(\theta^{*} / \theta_{1}\right)} \exp \left\{-\left[b_{0} \ln \left(\theta / \theta^{*}\right) / \ln \left(\theta^{*} / \theta_{1}\right)\right]^{2}\right\}
$$

Previsivelmente, o incremento na temperatura e deformação induzirão um estreitamento do espetro, bem como o deslocamento do mesmo para tempos mais curtos. De referir o parâmetro $b$, que é inversamente proporcional ao desvio padrão, do logaritmo dos tempos de relaxação (André et al., 2014), relacionado com bode acordo com a expressão

$$
b=b o l / \ln \left(\theta^{*} / \theta_{1}\right)
$$

\subsection{Ajuste Experimental do Modelo}

\subsubsection{Poli(metacrilato de metilo) - PMMA e poli(etileno tereftalato) - PET}

Da aplicação do modelo foram obtidos os parâmetros físicos ou propriedades para o poli(metacrilato de metilo) - PMMA (material não temperado sujeito a ensaio de relaxação de duração de 2,5 horas) e o poli(etileno tereftalato) - PET (material temperado sujeito a ensaio de relaxação de tensões de duração de 24 horas).

Tabela 1- Parâmetro/propriedade física para o PMMA e PET calculados com o modelo.

\begin{tabular}{lcc}
\hline Parâmetro/propriedade física & PMMA & PET \\
\hline$E_{0}(\mathrm{GPa})$ & $1,26(4)$ & $0,69(9)$ \\
\hline$E_{\infty}(\mathrm{MPa})$ & $1,00(0)$ & $77,40(2)$ \\
\hline$E_{\alpha, 1}(\mathrm{~kJ} / \mathrm{mol})$ & $35,65(5)$ & $79,43(3)$ \\
\hline$T_{c}(\mathrm{~K})$ & $455,20(3)$ & $412,74(6)$ \\
\hline$b_{0}(-)$ & $0,30(4)$ & $2,29(8)$ \\
\hline
\end{tabular}


A Tabela 1 apresenta os parámetros físicos ou propiedades para os dois polímeros em estudo, obtidos com o modelo, sendo os erros relativos inferiores a $1 \%$. Não considerando o efeito do escoamento viscoso, são de salientar os valores obtidos para os patamares do módulo. Para ambos os polímeros, o patamar do módulo de relaxação instantâneo, $E_{0}$, como era de prever, é à volta de $10^{9} \mathrm{~Pa}$. Por outro lado, o patamar do módulo para tempos longos, $E_{\infty}$, apresenta o valor $10^{6} \mathrm{~Pa}$ para o PMMA, valor típico dos polímeros amorfos. O valor é superior no PET, em virtude de, apesar de ter sido sujeito a tratamento térmico de tempera, ainda conter zonas cristalinas, que, como que aprisionando as zonas amorfas, conferem maior resistência ao polímero.

De igual modo é de relevar a temperatura de crossover, para ambos os polímeros, ser praticamente coincidente com a reportada na literatura (Donth, 2001). Sendo $T_{c} \sim 1,2 T_{g}$ para muitos materiais (Sillescu, 1999), o valor obtido pelo modelo para o PMMA é muito próximo do referido na literatura $\left((105+273,15)^{*} 1.2=453,78 \mathrm{~K}\right)$, bem como no caso do PET $((70+273,15) * 1,2=411,78 \mathrm{~K})$.

A Figura 1 ilustra o resultado do ajuste, através da aplicação da equação (1), das curvas experimentais, com a duração de 2,5 horas, do módulo de relaxação para o PMMA, às temperaturas de $40^{\circ} \mathrm{C}$ e $50 \stackrel{\circ}{ } \mathrm{C}$, e deformações de $4 \%$ e $5 \%$.

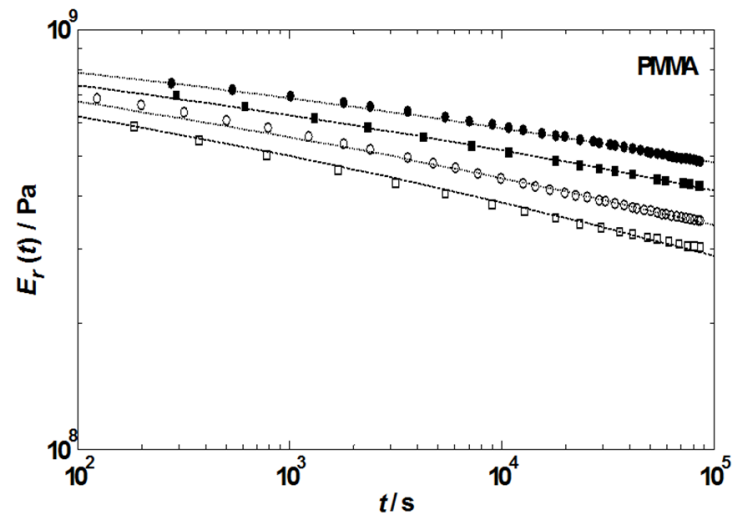

Figura 2 - Ajuste dos dados do módulo de relaxação para o PMMA, usando a equação (1): $\bullet 40{ }^{\circ} \mathrm{C}, 4 \%$; $440^{\circ} \mathrm{C}$, $5 \%$; $550^{\circ} \mathrm{C}, 4 \%$; $\square 50{ }^{\circ} \mathrm{C}, 5 \%$..

A Figura 2 ilustra o resultado do ajuste, através da aplicação da equação (1), das curvas experimentais, com a duração de 24 horas, do módulo de relaxação para o PET, previamente recozido à temperatura de 85 ㄷ, seguido de arrefecimento brusco em água a $60 \mathrm{C}$, ensaiado às temperaturas de $30^{\circ} \mathrm{C}$ (deformação a 3\% e 4\%) e $50^{\circ} \mathrm{C}$ (deformação de 3\%, 4\% e 5\%) 


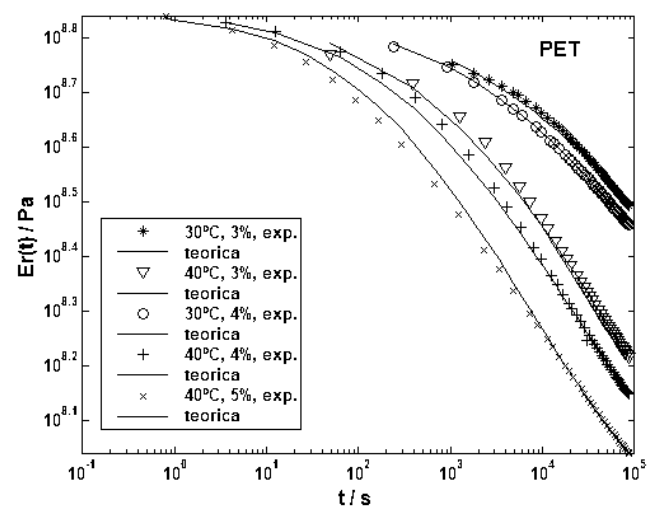

Figura 2 - Ajuste dos dados do módulo de relaxação para o PET a 30 e 40 oC, usando a equação (1).

Como era previsível, para os materiais estudados, o módulo de relaxação de tensões, para as deformações e temperaturas mais elevadas, surge para tempos inferiores em relação às deformações e temperaturas mais reduzidas (Figura 2).

A título ilustrativo da previsão do comportamento à relaxação de tensões para uma escala de tempos muito mais longa, apresenta-se a Figuras 3, para o PMMA às temperaturas de $40^{\circ} \mathrm{Ce}$ 50 ㄷ, e deformações de $4 \%$ e $5 \%$. De igual modo se apresentam os dados experimentais, com a duração de 2,5 horas, à temperatura de 50 C e $4 \%$.

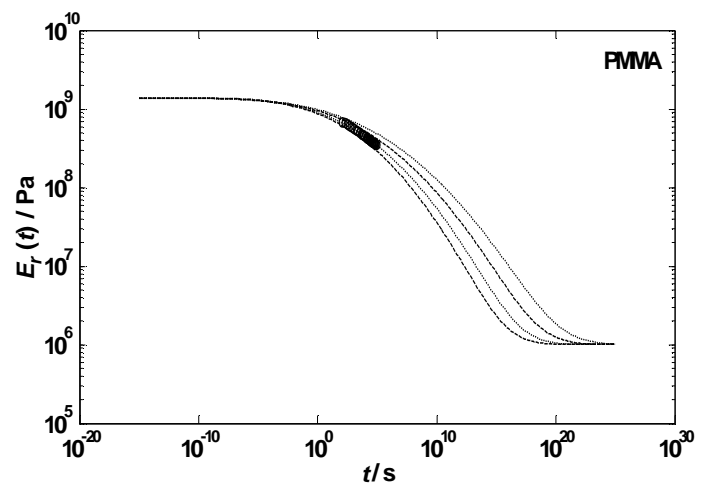

Figura 3 - Curvas previsionais de relaxação de tensões para o PMMA da direita para a esquerda: $40{ }^{\circ} \mathrm{C}, 4 \% ; 40$ ${ }^{\circ} \mathrm{C}, 5 \% ; 50{ }^{\circ} \mathrm{C}, 4 \% ; 50{ }^{\circ} \mathrm{C}, 5 \%$; $\circ$ dados experimentais a $50{ }^{\circ} \mathrm{C}, 4 \%$.

Na Figura 4, apresenta-se a previsão do comportamento à relaxação de tensões para uma escala de tempos muito mais longa, para o PET às temperaturas de $30^{\circ} \mathrm{C}$ (deformações de $3 \%$ e $4 \%$ ) e 40 oC (deformações de $3 \%, 4 \%$ e $5 \%$ ). De igual modo se apresentam os dados experimentais, com a duração de 24 horas. 


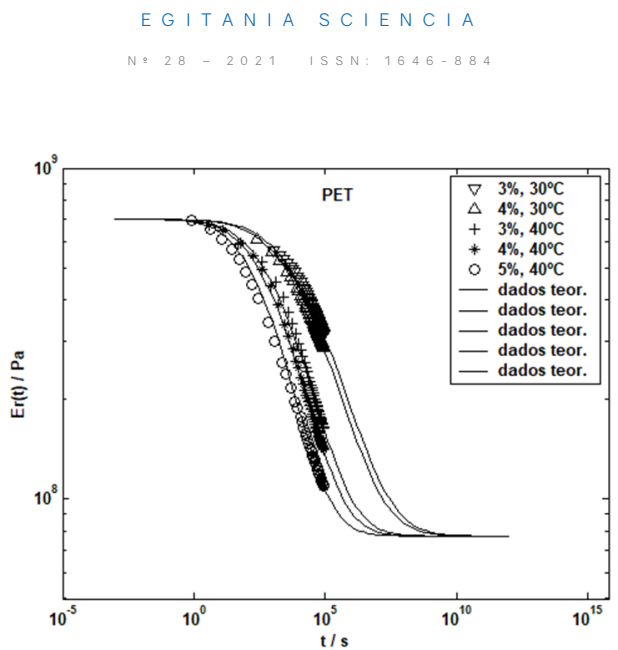

Figura 4 - Curvas previsionais de relaxação de tensões obtidas para o PET, a $30{ }^{\circ} \mathrm{C}$ (deformações de $3 \%$ e $4 \%$ ) e $40{ }^{\circ} \mathrm{C}$ (deformações de $3 \%, 4 \%$ e $5 \%$ ), e os dados experimentais.

Com base em ensaios de curta duração de 2,5 horas (PMMA) e de 24 horas (PET), o valor preditivo do presente modelo (equação 1) é particularmente bem ilustrado na sua capacidade de prever quantitativamente características do comportamento experimentalmente acessíveis só para tempos muito mais longos, como são o módulo de relaxação de tensões para tempo infinito (para polímeros amorfos e semi-cristalinos), assim como os próprios tempos de relaxação.

Para tempos muito curtos, logo após a deformação inicial, os segmentos moleculares não tiveram tempo de se reorientar significativamente, sendo elevado o valor do módulo (o primeiro patamar próximo de $10^{9} \mathrm{~Pa}$ na Figura 4), o que corresponde a terem-se forçado as ligações covalentes e os ângulos de valência das cadeias moleculares.

Para tempos muito longos, a reorientação molecular teve tempo de ocorrer, pelo que a deformação imposta não tem que ser suportada pelas ligações covalentes e ângulos de valência, como sucedia para tempos muito curtos, sendo por isso mais baixo o valor do módulo. Como o valor do módulo previsto pelo modelo (ver Figura 4) para tempos longos é superior a $10^{6} \mathrm{~Pa}$, indicia que, pese embora o tratamento térmico sofrido, o PET comporta-se como polímero semi-cristalino. As cadeias moleculares desenrolam-se, mas existem pontos de prisão que são as zonas cristalinas, provocando o aumento do módulo, relativamente ao módulo característico dos polímeros amorfos, $10^{6} \mathrm{~Pa}$.

A Figura 5, resulta da aplicação da equação 2 do modelo, é apresentada com o objetivo de descrever o efeito da deformação $\left(3,4\right.$ e $5 \%$ ) e da temperatura $\left(30\right.$ e $\left.40{ }^{\circ} \mathrm{C}\right)$ na largura dos espectros dos tempos de relaxação do PET, sendo aqueles tempos característicos de cada tipo de movimento molecular que contribui para a resposta mecânica do material, e são inversamente proporcionais à frequência dos movimentos correspondentes (a qual por sua vez é função da temperatura). 


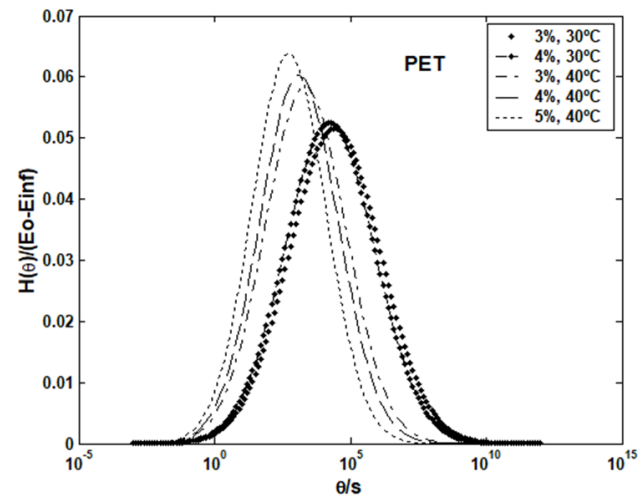

Figura 5: Espectro dos tempos de relaxação para o PET, para a temperatura de 30 oC (deformações de 3 e $4 \%$ ) e para a temperatura de 40 oC (deformações de 3, 4 e $5 \%$ ).

Tabela 2 - Valores do parâmetro $b$ em função da temperatura e da deformação para o PET, com base no modelo.

\begin{tabular}{cccc}
\hline $\mathrm{T}(\mathrm{o} \mathbf{C})$ & $\mathbf{3} \%$ & $\mathbf{4 \%}$ & $\mathbf{5 \%}$ \\
\hline 30 & 0,1827 & 0,1861 & \\
\hline 40 & 0,2069 & 0,2135 & 0,2262 \\
\hline
\end{tabular}

Na Tabela 2 encontra-se sumarizada a variação do parâmetro $b$ sob o efeito da temperatura e da deformação aplicada durante o ensaio de relaxação de tensões para o PET, com base no modelo desenvolvido.

Da observação dos espectros (Figura 5) para as várias deformações e duas temperaturas e da Tabela 2, é notório que o aumento da deformação e da temperatura, para além de induzirem a redução da largura do espetro (aumento do parâmetro $b$, que varia inversamente com a largura do espetro, ver equação (3)), deslocam o espectro dos tempos de relaxação para tempos mais curtos (reflexo da diminuição prevista no modelo dos tempos de relaxação médio, $\theta^{*}$, e mínimo, $\theta_{1}$ ).

\section{CONCLUSÕES}

1. O modelo, não considerando o escoamento viscoso, descreve a relaxação não linear de tensões para os polímeros PET e PMMA variando a temperatura e deformação, com erros relativos inferiores a $1 \%$, permitindo rápida extrapolação para diferentes temperaturas, escalas de tempo muito maiores e diferentes deformações, bem como determina os patamares inicial e final do módulo de relaxação de tensões.

2. O modelo desenvolvido considera a forma aproximada dos espectros dos tempos de relaxação, que preveem quantitativamente a sua evolução em função da temperatura e da deformação aplicada. 
3. As variações com a temperatura e com a deformação dos parâmetros que definem a forma do espectro ( $b_{0}, \tau_{1}$ e $\left.\tau^{*}\right)$ são as fisicamente esperadas e também elas matematicamente formuláveis em relações simples dedutíveis dos fundamentos do modelo.

4. O modelo desenvolvido (naturalmente, após prévia validação experimental e quantificação de parâmetros) é fisicamente descritivo e efetivamente preditivo, e não apenas semi-empírico, interpolativo e (algo mais deficientemente) extrapolativo, como as formulações alternativas conhecidas (Brostow, et al., 1993; Blonsky et al., 1994; Warren, et al., 2007; Brostow, 2009; Reis et al., 2019; Warren, et al., 2010). O valor preditivo do presente modelo é particularmente bem ilustrado na sua capacidade de prever quantitativamente características do comportamento experimentalmente quase inacessíveis, como é a relaxação de tensões para tempo infinito (para polímeros amorfos e semi-cristalinos).

5. A formulação é inteiramente analítica e os cálculos são extremamente rápidos num comum computador de secretária para tratar conjuntos completos de dados experimentais com diferentes temperaturas e deformações, permitindo extrapolar curvas de relaxação de tensões (até escalas de tempo indefinidamente longas) e calcular valores otimizados para todos os parâmetros físicos.

\section{REFERÊNCIAS}

André, J.R.S., (2004). Fluência de Polímeros - Fenomenologia e Modelação Dinâmica Molecular, dissertação de Doutoramento, Universidade de Aveiro, Aveiro.

André, J.R.S., Cruz Pinto, J.J.C., (2014). Modeling nonlinear stress relaxation of polymers, Polymer Engineering and Science, Vol. 54, Issue 2, 404-416, doi:10.1002/pen.23581.

Cruz Pinto, J.J.C., André, J.R.S., (2016). Towards the Accurate Modeling of Amorphous Non-Linear Materials - Polymer Stress Relaxation (I), Polymer Engineering and Science, Vol. 56, Issue 3, 348360, doi: 10.1002/pen.24260.

Blonski, S. Brostow, W. Kubát, J. (1994). Molecular-dynamics simulations of stress relaxation in metals and polymers. Physical Review B, Vol. 49, Issue 10, 6494-6500.

Brostow, W. Kubát, J. (1993). Molecular-dynamics simulation of stress relaxation on a triangular lattice. Physical Review B, Vol. 47, Issue 13, 7659-7667.

BROSTOW, W. (2009). Reliability and prediction of long-term performance of polymer-based materials. Pure and Applied Chemistry, Vol. 81, Issue 3, 417-432, DOI: 10.1351/PAC-CON-08-0803

Donth, E. (2001). The Glass Transition: Relaxation Dynamics in Liquids and Disordered Materials, Springer-Verlag, Berlin Heidelberg.

Findley, W. Lai, J.S. Onaran, K. (1989) Creep and Relaxation of Non-Linear Viscoelastic Materials, Dover Publications, New York.

Högfors, Ch. Kubát, J. Rigdahl, M. (1981). A cooperative model of stress relaxation in solids. physica status solidi., Vol. 107, Issue 1, 147-155.

Houshyar, S. Shanks, R.A. Hodzic, A. (2005) Tensile creep behaviour of polypropylene fibre reinforced polypropylene composites, Polymer Testing, Vol. 24, Issue 2, 257-264.

Kubát, J. (1979). Stress relaxation in solid polymers. Macromolecular Chemistry Suppl., Vol. 3, Issue S19791, 233-245.

Kubát, J. (1982). A cooperative mechanism limiting the spectral width of stress relaxation processes in solids. PHYSICA STATUS SOLIDI, Vol 111, Issue 2, 599-606. 
Reis,P.N.B, Silva, M.P., Santos, P., (2019) Stress Relaxation in Delaminated Carbon/Epoxy Composites. FIBERS AND POLYMERS, VOL.20, Issue.6, 1284-1289, DOI 10.1007/s12221-0198916-x.

Sillescu, H. J. (1999). Heterogeneity at the glass transition: a review. Journal of Non-Crystalline Solids, Vol. 24381-108.

Sweeney, J., Ward, I.M., (1990) A unified model of stress relaxation and creep applied to oriented polyethylene. JOURNAL OF MATERIALS SCIENCE 25 (1990) 697-705.

Tobolsky, A. V. (1960). Properties and Structure of Polymers, Wiley, New York.

Warren, M., Rottler, J. (2007). Simulations of aging and plastic deformation in polymer glasses. Physical. Review E, Vol. 76, Issue 3, 031802-031811. http://dx.doi.org/10.1103/PhysRevE.76.031802.

Warren, M., Rottler, J. (2010). Microscopic View of Accelerated Dynamics in Deformed Polymer Glasses. Physical Review Letters, Vol. 104, Issue 20, 205501-205505. http://dx.doi.org/10.1103/ PhysRevLett.104.205501. 
